\title{
Tailoring barocaloric and magnetocaloric properties in low-hysteresis magnetic shape memory alloys
}

\author{
Enric Stern-Taulats ${ }^{\mathrm{a}}$, Antoni Planes ${ }^{\mathrm{a}}$, Pol Lloveras ${ }^{\mathrm{b}}$, Maria Barrio $^{\mathrm{b}}$, \\ Josep-Lluís Tamarit ${ }^{\mathrm{b}}$, Sabyasachi Pramanick ${ }^{\mathrm{c}}$, Subham Majumdar ${ }^{\mathrm{c}}$, \\ Suheyla Yüce ${ }^{\mathrm{d}}$, Baris Emre ${ }^{\mathrm{e}}$, Carlos Frontera ${ }^{\mathrm{f}}$, Lluís Mañosa ${ }^{\mathrm{a}}$ \\ ${ }^{a}$ Departament d'Estructura i Constituents de la Matèria, Facultat de Física, Martí $i$ \\ Franquès 1, Universitat de Barcelona, 08028 Barcelona, Catalonia. \\ ${ }^{b}$ Departament de Física i Enginyeria Nuclear, ETSEIB, Diagonal 647, Universitat \\ Politècnica de Catalunya, 08028 Barcelona, Catalonia. \\ ${ }^{c}$ Department of Solid State Physics, Indian Association for the Cultivation of Science, \\ Jadavpur, Kolkata 700032, India. \\ ${ }^{d}$ Department of Physics, Science and Literature Faculty, Ondokuz Mayis University, \\ Kurupelit, 55139 Samsun, Turkey \\ ${ }^{e}$ Faculty of Engineering, Department of Engineering Physics, Ankara University, 06100 \\ Besevler, Ankara, Turkey. \\ ${ }^{f}$ Institut de Ciència de Materials de Barcelona, Campus UAB, Bellaterra, Catalonia.
}

\begin{abstract}
We report on the barocaloric and magnetocaloric effects in a series of low-hysteresis Ni-Mn-In magnetic shape memory alloys. We show that the behaviour exhibited by several quantities that characterise these caloric effects (isothermal entropy change, adiabatic temperature change and refrigerant capacity) can be rationalised in terms of the relative distance between the Curie point of the austenite and the martensitic transition temperature. It is found that the two caloric effects exhibit opposite trends. The behaviour of the barocaloric effect parallels that exhibited by the transition entropy change, thereby showing larger values for weakly magnetic samples. Regarding the magnetocaloric effect, the entropy change is maximum for those samples transforming martensitically close to the Curie point of the austenite. Such a maximum value does not correspond to the maximum adiabatic temperature change, and samples with martensitic transition slightly below the Curie point do have larger temperature changes as a result of the strongest sensitivity of the transition to the magnetic field.
\end{abstract}

Keywords: barocaloric and magnetocaloric effects; magnetic shape 
memory; martensitic transformation

\section{Introduction}

Caloric effects refer to the isothermal entropy change or to the adiabatic temperature change exhibited by a material under the application or removal of a given external field [1]. These effects are enhanced near to phase transitions $[1,2,3]$, and particularly appealing are first-order phase transitions where the latent heat of the transition provides a large contribution to the field-induced entropy change and the effect is considered to be giant. Up to now, giant caloric effects have been reported in a broad variety of materials under the application of magnetic field (magnetocaloric effect) $[4,5]$, electric field (electrocaloric effect) [6,7], hydrostatic pressure (barocaloric effect) $[8,9]$ and uniaxial stress (elastocaloric effect) [10, 11].

The potential use of giant caloric materials in cooling devices requires large values for the field-induced entropy changes which prompts materials with first-order phase transitions as the best candidates for this application. There are, however, other factors affecting the caloric performances of a given material. On the one hand hysteretic effects typically associated with first order phase transitions critically compromise the reproducibility of the caloric effect under field cycling. On the other hand, a strong sensitivity of the transition temperature to the external field will result in large values for the adiabatic temperature change and a broadening of the temperature window where giant (reversible) caloric effects occur. According to Clausius-Clapeyron equation, the field dependence of the transition temperature weakens as the entropy change increases (provided that the change of the property conjugated to the applied field remains constant). Therefore, the optimal caloric performances of a given material will result from the best balanced tuning of all these parameters. In the present paper we have addressed these issues for the case study of magnetic shape memory alloys.

Ni-Mn-based magnetic shape memory alloys undergo a martensitic transition from a cubic high temperature phase with a Heusler structure (austenite) towards a low temperature close-packed phase (martensite) [12] whose crystal structure depends on composition [13]. The martensitic transition is first order, with a lattice distortion dominated by a shear strain with a relatively large volume change, and encompasses a large latent heat. There is a strong coupling between magnetic and structural degrees of freedom in these alloys 
[14] and changes in strain and volume are accompanied by changes in magnetization. These changes make the martensitic transition in these materials to be sensitive to uniaxial stress, hydrostatic pressure, and magnetic field, and giant elastocaloric [15, 16], barocaloric [8] and magnetocaloric [17, 18] effects have already been reported for these alloys.

As previously mentioned, giant caloric effects are typically associated with a large latent heat at the first-order phase transition which results in a large transition entropy change $\left(\Delta S_{t}\right)$. In magnetic shape memory alloys there are two major contributions to $\Delta S_{t}$ : structural and magnetic. The structural contribution is mainly due to lattice vibrations (phonons) and stabilizes the high temperature cubic phase while the magnetic contribution stabilizes the low temperature martensitic phase (which is magnetically more disordered) [19]. While the structural contribution is almost constant within a broad temperature range, the magnetic contribution does depend on how far the martensitic transition temperature is from the Curie point of the austenitic phase. Actually, it has been found that the value of $\Delta S_{t}$ can be tailored by tuning the relative distance between the Curie point and the martensitic transition[20, 21].

The martensitic transition occurs with hysteresis which mostly originates from elastic incompatibilities at the habit plane that separates the untransformed (austenite) from the transformed (martensite) regions. By using the crystallographic theory of martensite it has been shown that the alloy composition can be tuned so that the crystal structures of both phases meet certain geometric compatibilities and the hysteresis is drastically reduced which results in an enhanced reversibility of the martensitic transition [22, 23]. By considering these elastic compatibility conditions magnetic shape memory alloys with hysteresis around $6 \mathrm{~K}$ and $4 \mathrm{~K}$ have already been produced [24, 25].

In the present paper we have studied the barocaloric and magnetocaloric properties of magnetic shape memory alloys. We have investigated the $\mathrm{Ni}$ Mn-In system which has been shown to exhibit the strongest sensitivity of the transition temperatures to both magnetic field and hydrostatic pressure [26]. The relative distance between the martensitic transition and the Curie point of the cubic phase has been tailored by tuning the composition of the samples. Furthermore, the compositions have been selected in such a way that the martensitic transition takes place with a very narrow hysteresis. Calorimetry under magnetic field and under hydrostatic pressure has been used to study the caloric response of these samples. 


\section{Experimental details}

Polycrystalline Ni-Mn-In samples were prepared by arc-melting the pure metals under an argon atmosphere in a water-cooled $\mathrm{Cu}$ crucible. To improve homogeneity, the samples were remelted several times and, subsequently, the ingots were vacuum sealed in a quartz tube and annealed at $900^{\circ} \mathrm{C}$ for $48 \mathrm{~h}$ followed by quenching in ice water. Specimens for magnetization (typically $\sim 50 \mathrm{mg}$ ), for calorimetry under magnetic field (typically $\sim 150 \mathrm{mg}$ ) and for calorimetry under hydrostatic pressure (typically $\sim 1 \mathrm{~g}$ ) were cut with a diamond saw. The final composition of the samples was determined by EDX.

Magnetization was measured by using a superconducting quantum interferometer device (SQUID, Quantum design). Measurements were done under an applied field of 50 Oe, in cooling-heating cycles between 240 and $400 \mathrm{~K}$ at a rate of $2 \mathrm{~K} / \mathrm{min}$.

Calorimetry under magnetic field was carried out using a custom-built calorimeter capable of operating under external magnetic fields, described in Ref. [27]. Cooling-heating cycles were performed at a rate $0.5 \mathrm{~K} / \mathrm{min}$ at isofield conditions for magnetic fields in the range 0-6 T. Isothermal calorimetric runs were also performed while the magnetic field was cyclically swept between 0 and $6 \mathrm{~T}$ at a rate $0.16 \mathrm{~T} / \mathrm{min}$. Hydrostatic pressure calorimetry was carried out on a second custom-built calorimeter described in Ref. [8]. In that device, the thermal signal was measured by a chromel-alumel thermocouple embedded into the sample. The calorimeter under magnetic field features a better accuracy than the one under hydrostatic pressure and therefore it is used to determine the thermal properties (transition temperatures, and entropy changes) associated with the martensitic transition at atmospheric pressure and in the absence of magnetic field.

\section{Experimental results}

For each of the studied samples, EDX analysis were performed on three separate locations of the sample surface, and sample compositions listed in Table 1 correspond to averaged values over these three measurements. The samples are close to the composition line $\mathrm{Ni}_{50} \mathrm{Mn}_{50-x} \mathrm{In}_{x}$ with the exception of sample B which is slightly richer in Ni and Mn deficient. This sample has also been included into the study to reinforce the finding that the relevant parameter which controls many caloric performances is the reduced distance between martensitic and Curie temperatures, as will be shown later. 
Table 1: Sample composition, forward $\left(T_{M}\right)$ and reverse $\left(T_{A}\right)$ martensitic temperatures, thermal hysteresis $\left(T_{A}-T_{M}\right)$, Curie temperature $\left(T_{c}\right)$, transition entropy change $\left(\Delta S_{t}\right)$, magnetic field dependence of the martensitic transition temperature $\frac{d T}{\mu_{0} d H}$, pressure dependence of the martensitic transition temperature $\frac{d T}{d p}$, and effective magnetic moment per formula unit $\mu$.

\begin{tabular}{llllllllll}
\hline Sample & Composition & $\begin{array}{l}T_{M} \\
(\mathrm{~K})\end{array}$ & $\begin{array}{l}T_{A} \\
(\mathrm{~K})\end{array}$ & $\begin{array}{l}T_{A}-T_{M} \\
(\mathrm{~K})\end{array}$ & $\begin{array}{l}T_{c} \\
(\mathrm{~K})\end{array}$ & $\begin{array}{l}\Delta \mathrm{S}_{t} \\
(\mathrm{~J} / \mathrm{kg} \mathrm{K})\end{array}$ & $\begin{array}{l}\frac{d T}{\mu_{0} d H} \\
(\mathrm{~K} / \mathrm{T})\end{array}$ & $\begin{array}{l}\frac{d T}{d p} \\
(\mathrm{~K} / \mathrm{kbar})\end{array}$ & $\begin{array}{l}\mu \\
\left(\mu_{B}\right)\end{array}$ \\
\hline $\mathrm{A}$ & $\mathrm{Ni}_{50.5} \mathrm{Mn}_{34.1} \operatorname{In}_{15.4}$ & 346 & 350 & 4 & 304 & 39.9 & -0.12 & 1.83 & 4.79 \\
$\mathrm{~B}$ & $\mathrm{Ni}_{51.3} \mathrm{Mn}_{32.5} \mathrm{In}_{16.2}$ & 329 & 333 & 4 & 298 & 40.8 & -0.26 & 1.65 & 4.25 \\
$\mathrm{C}$ & $\mathrm{Ni}_{49.8} \mathrm{Mn}_{34.2} \mathrm{In}_{16.0}$ & 329 & 333 & 4 & 307 & 40.3 & -0.42 & 1.95 & 5.93 \\
$\mathrm{D}$ & $\mathrm{Ni}_{49.8} \mathrm{Mn}_{33.4} \mathrm{In}_{16.8}$ & 306 & 309 & 3 & 302 & 32.0 & -1.37 & 1.37 & 5.13 \\
$\mathrm{E}$ & $\mathrm{Ni}_{50.0} \mathrm{Mn}_{33.1} \mathrm{In}_{16.9}$ & 275 & 280 & 5 & 307 & 24.0 & -2.64 & 1.88 & 4.80 \\
\hline
\end{tabular}

Figure 1a shows low field magnetization as a function of temperature for the studied samples. The inset shows a magnified view which illustrates the magnetization change at the martensitic transition for samples A, B and $\mathrm{C}$, for which the austenite is paramagnetic. By contrast, for sample E, the martensitic transition occurs well below the Curie point of the austenite. For sample D both martensitic and magnetic transitions are almost coincident. For all samples the low field magnetization in martensite is lower than in austenite. Figure $1 \mathrm{~b}$ shows the inverse of the magnetic susceptibility $\left(\chi^{-1}\right)$ as a function of temperature. Data exhibit a good linear behaviour within the temperature range corresponding to the paramagnetic state of austenite. Linear fit to the data enabled us to determine the paramagnetic Curie point $\left(T_{c}\right)$ and the effective magnetic moment $\mu$ which are listed in Table 1 for all samples. Within experimental errors no systematic dependence upon sample composition has been found for these quantities. The values found for $\mu$ are consistent with those reported for magnetic shape memory alloys with compositions close to those of the samples studied here [28] but they are slightly lower than the magnetic moments obtained from high field magnetization measurements [29] and from first principle calculations [30, 31]. Such a difference may be due to the presence of antiferromagnetic correlations [32]. On the other hand, the paramagnetic Curie temperatures determined for our samples are in good agreement with the Curie points reported for $\mathrm{Ni}_{50} \mathrm{Mn}_{50-x} \operatorname{In}_{x}[29]$.

Illustrative examples of the calorimetric curves obtained under constant hydrostatic pressure and under constant applied magnetic field are presented in figure 2. From the calorimetric curves we have computed forward $\left(T_{M}\right)$ and reverse $\left(T_{A}\right)$ transition temperatures which correspond, respectively, to 
the temperatures where $50 \%$ of the sample has transformed to martensite on cooling, and to austenite on heating. The thermal hysteresis is computed as the difference between these two temperatures and the data are compiled in Table 1. It is noticeable that for all the samples hysteresis is in the range 3-5 K, which is a significantly low value for martensitic transformations [23]. Interestingly, the lower value is found for the sample with the martensitic transition closest to the Curie point (sample D), as previously reported in other alloys $[24,25]$ which points to the fact that the maximal lattice compatibility is achieved in those alloys with martensitic and Curie transitions very close to each other. From numerical integration of the calorimetric curves we have obtained the transition entropy change $\Delta S_{t}$. Values listed in Table 1 correspond to the data obtained at atmospheric pressure and at zero magnetic field, and are an average over cooling and heating values. Application of hydrostatic pressure shifts the transition towards higher temperatures (fig. 2 , left panels) which indicates an enhancement in the stability of the martensite which has a lower unit cell volume [33] while application of a magnetic field, shifts the martensitic transition towards lower temperatures (fig. 2, right panels), which is consistent with magnetic field stabilizing the austenite phase which displays a larger magnetization than martensite. The hydrostatic pressure and magnetic magnetic field dependences of the temperatures $T_{M}$ and $T_{A}$ are plotted in figures $3 \mathrm{a}$ and $3 \mathrm{~b}$. Within experimental errors the increase of $T_{M}$ and $T_{A}$ with pressure and the decrease with magnetic field are found to be linear. We have quantified the shift in the martensitic transition with hydrostatic pressure and magnetic field by averaging the values of the slopes of the lines in figure 3 obtained on cooling and heating runs for each sample. The corresponding data are listed in Table 1.

\section{Discussion}

\subsection{Isothermal entropy changes}

As described in refs. [9, 27], for each fixed value of the external parameter $Y$ (where $Y$ corresponds to either hydrostatic pressure $p$ or magnetic field $H$ ), the entropy (referenced to a given state at a temperature $T_{0}$ far from the martensitic transition) is obtained as

$$
S(T, Y)-S\left(T_{0}, Y\right)=\int_{T_{0}}^{T} \frac{1}{T} \frac{\dot{Q}(Y, T)}{\dot{T}} d T
$$


where $\dot{Q}$ and $\dot{T}$ are the heat flux and cooling (or heating) rate, respectively. The corresponding isothermal entropy change accounting for the caloric effect is then obtained (quasi-direct method) by subtraction of the entropy curves computed using Eq. 1 as:

$$
\Delta S \equiv S(T, Y)-S(T, 0)
$$

Results found for the BCE and MCE are shown in Figure 4. Since pressure stabilizes the martensitic phase (as cooling does), the pressure induced entropy change in fig. 4 has been computed from calorimetric cooling curves. On the other hand, as magnetic field stabilizes the austenitic phase (as heating does), entropy values have been computed from heating calorimetric curves.

For all the samples, BCE is found to be conventional (entropy decreases when the sample is isothermally compressed) and MCE is found to be inverse (entropy increases upon isothermal application of magnetic field). The absolute values of $\Delta S$ increase upon increasing hydrostatic pressure and magnetic field as shown in figure 5 . It is worth remarking that present results data for the pressure induced entropy change are the highest $\Delta S$ values reported for any magnetic alloy until now [8, 9? , 33, 34, 35, 36].

In view of possible applications of $\mathrm{BCE}$ and $\mathrm{MCE}$ effects in refrigerating devices it is crucial to determine the repeatability of the entropy values upon successive pressure and magnetic field cycles. A direct determination of such a repeatability can be obtained by isothermal calorimetric measurements while the external parameter (pressure or magnetic field) is cyclically varied. While this kind of measurements can be performed with the calorimeter under magnetic field, pressure cycles cannot be carried out in a regular controlled manner. However, a good estimation of the reversibility of a caloric effect can be gained from the quasi-direct method by analysing the overlap of the $\Delta S$ vs. $T$ curves obtained during heating and cooling runs [25, 35]. In the left panels of figure we have plotted the BCE for $2.5 \mathrm{kbar}$ (except for sample $\mathrm{D}$ for which the maximum pressure was $2 \mathrm{kbar}$ ) and in the right panels of figure 6 we have plotted the MCE for a magnetic field of $6 \mathrm{~T}$. The expected entropy values for cyclic variation of hydrostatic pressure and magnetic field are depicted as light grey lines, and the temperature region where reversibility is expected corresponds to the shaded region. To confirm the reliability of the method in determining the repeatability of $\mathrm{BCE}$ and $\mathrm{MCE}$ we have performed isothermal calorimetric measurements for samples D and 
E while magnetic field was cyclically varied between 0 and $6 \mathrm{~T}$. The obtained entropy values are plotted as symbols in Figure 6, where solid symbols stand for the first application (or removal) of the magnetic field and open symbols stand for the successive application (or removal) of the field. As shown in the figure there is a good coincidence between data derived from the quasi-direct method and those obtained for the direct method. While the reproducibility of the BCE is small for all samples, significant repeatable values for $\Delta S$ are obtained at the MCE of samples D and E over a temperature span of several degrees.

\subsection{Adiabatic temperature changes}

The adiabatic temperature changes associated with the BCE and MCE can be indirectly determined by the proper combination of DSC under external field and specific heat $(C)$ measurements $[37,38]$. In this case a good approximation is to assume that beyond the transition region $C$ can be considered to be independent from the external field [38]. Furthermore, for magnetic shape memory alloys, the specific heat in both martensite and austenite does not significantly depend on composition [38, 19, 39]. We have measured $C$ for one of the studied Ni-Mn-In samples (sample D) by using a modulated differential scanning calorimeter (Q-2000) from TA instruments. Results are in good agreement with those reported for other magnetic shape memory alloys $[28,38,19,39]$. We have combined these data with the entropy vs. temperature curves obtained at different values of hydrostatic pressure and magnetic field to compute the pressure induced and magnetic field induced adiabatic temperature changes shown in figure 7 .

The adiabatic application of hydrostatic pressure increases the temperature of the sample (in concordance with the conventional nature of the $\mathrm{BCE}$ ) while application of magnetic field decreases the temperature of the sample (in agreement with the inverse nature of the MCE). Regarding MCE, $\Delta T$ exhibits very tiny values for those samples with the martensitic transition temperature above the Curie point, but for samples transforming below the Curie point $\Delta T$ reaches values significantly large (6-8 $\mathrm{K}$ at $6 \mathrm{~T})$, which are slightly above those recently reported $(7 \mathrm{~K})$ for a Ni-Mn-In alloy of close composition at $6 \mathrm{~T}[38]$. On the other hand, the $\mathrm{BCE}$ exhibits larger $\Delta T$ values for those samples with the martensitic transition in the paramagnetic state, with values around $3-4 \mathrm{~K}$ (at $2.5 \mathrm{kbar}$ ). 


\subsection{Tuning caloric performances}

The large changes in entropy in giant caloric materials, are associated with the latent heat (and thereby to the transition entropy change $\Delta S_{t}$ ) of a first-order phase transition. In magnetic shape memory alloys, it has been shown that $\Delta S_{t}$ scales with the relative distance between the martensitic transition and the Curie point of the autenite $\left(T_{c}-T_{M}\right) / T_{c}[20,21]$. In figure 8a we have plotted $\Delta S_{t}$ (at atmospheric pressure and zero magnetic field) for the studied samples as a function of $\left(T_{c}-T_{M}\right) / T_{c}$ (solid green squares). It is shown that for $T_{M}>T_{c}, \Delta S_{t}$ tends towards a constant value, while it begins to decrease as $T_{M}$ approaches $T_{c}$. This behaviour is a consequence of the opposite contributions from the magnetic and vibrational degrees of freedom to the transition entropy change: the vibrational entropy in martensite is lower than in austenite, with a value which does not depend on composition. Hence, for those samples transforming martensitically from a paramagnetic austenite, the magnetic contribution is very small and $\Delta S_{t}$ is dominated by the vibrational contribution which is constant. However, for $T_{M} \leq T_{c}$, the magnetic contribution becomes relevant, with the magnetic entropy of martensite being larger than that of austenite. Hence, as $T_{M}$ gets lower than $T_{c}$ the magnetic entropy increases in magnitude thereby leading to a decrease in $\Delta S_{t}$. Eventually for low enough $T_{M}$ the magnetic term balances the vibrational one resulting in a vanishing transition entropy change and the martensitic transition no longer occurs [40, 41].

The scaling of $\Delta S_{t}$ points to the fact that the relative distance between martensitic and Curie points can also be a good parameter to rationalize the several quantities accounting for the caloric effects in magnetic shape memory alloys. In that case, the shift in the martensitic transition with hydrostatic pressure and with magnetic field must be taken into account, and the actual $T_{M}$ values that have to be used in computing $\left(T_{c}-T_{M}\right) / T_{c}$, are those measured at the corresponding values of $p$ and $H$. Isothermal entropy changes for the $\mathrm{BCE}$ (red triangles) and MCE (blue circles) are plotted in figure 8a as a function of $\left(T_{c}-T_{M}\right) / T_{c}$. For BCE, the maximum $\Delta S$ data correspond to an applied pressure of $2.5 \mathrm{kbar}$, and for MCE, maximum $\Delta S$ data correspond to a magnetic field of $6 \mathrm{~T}$ (blue circles). The entropy change corresponding to the BCE parallels the behaviour of $\Delta S_{t}$, showing larger and constant values for $T_{M}>T_{c}$ and decreasing for $T_{M} \leq T_{c}$. The fact that the entropy values for BCE are lower than $\Delta S_{t}$ reflects than the applied pressures are not large enough to accomplish transformation of the full sample. Interestingly, the entropy values for the MCE exhibit a behaviour different from that of $\Delta S_{t}$. 
For $T_{M}>T_{c}$, MCE exhibits low entropy values, as expected from the weak magnetism in this region (see figure 1). A maximum field-induced entropy change is attained when the martensitic transition occurs close to the Curie point of the austenite $\left(T_{M} \sim T_{c}\right)$, and for $T_{c}>T_{M}$ it decreases again because of the decrease in $\Delta S_{t}$.

We have also plotted the maximum adiabatic temperature change for BCE (red triangles) and MCE (blue circles) as a function of $\left(T_{c}-T_{M}\right) / T_{c}$ in figure $8 \mathrm{~b}$. Data correspond to a hydrostatic pressure of $2.5 \mathrm{kbar}$ (except for sample $\mathrm{D}$ which corresponds to $2 \mathrm{kbar}$ ) and to a magnetic field of $6 \mathrm{~T}$ (blue circles). Again, the behaviour of $\Delta T$ for $\mathrm{BCE}$ is similar to the behaviour of $\Delta S_{t}$. However, for the MCE, $\Delta T$ does not follow the trends of the magneticfield induced entropy change: as expected it shows low values for weak magnetic samples, but it raises steadily as $T_{M}$ becomes lower than $T_{c}$. Actually, it has to be taken into account that for a given caloric effect, the adiabatic temperature change does not only depend on the entropy change but also on how sensitive the transition temperature is to the applied external field. As shown in Table $1, d T / d p$ can be considered to be constant within the studied range. On the other hand, a significant increase is observed in $d T / d H$. Such an increase is understood in terms of the Clausius-Clapeyron equation and is a consequence of the larger values of the magnetization change across the martensitic transition arising from the larger ferromagnetic order of the austenite as $T_{M}$ becomes lower and lower than $T_{c}$ (see fig. 1). Inspection of figures $8 \mathrm{a}$ and $8 \mathrm{~b}$ shows that for the MCE although the entropy change decreases at large $T_{c}-T_{M}$ values, the adiabatic temperature change is large due to the strong sensitivity of the transition temperature to the magnetic field (see Table 1).

It is finally worth analysing the behaviour of the Refrigerant Capacity $(R C)$ which we have have computed as $R C=|\Delta S|_{\max } \times \delta T$, where $\delta T$ is the full width at half maximum for the $\Delta S$ vs $T$ curves (Figure 4). Data for a hydrostatic pressure of $2.5 \mathrm{kbar}$ (red triangles) and a magnetic field of $6 \mathrm{~T}$ (blue circles) are shown in fig. 8c. For the BCE, weakly magnetic samples exhibit the larger $R C$, in concordance with the trends exhibited by both $\Delta S$ and $\Delta T$. On the other hand, for MCE, although samples with martensitic and Curie points close each other have the largest entropy change, the $R C$ is significantly larger for samples with the martensitic transition well below the Curie point, due to the strongest sensitivity of the transition temperature to magnetic field which results in a broader temperature span. 


\section{Summary and conclusions}

We have studied the barocaloric and magnetocaloric effects in a series of composition related Ni-Mn-In magnetic shape memory alloys by means of calorimetry under hydrostatic pressure and magnetic field. We have tailored the composition of the alloys in such a way that all samples exhibit a martensitic transition with a very narrow hysteresis of 3-5 K. We have shown that the relative distance between the martensitic transition and the Curie point of the austenite is a good parameter to rationalise the behaviour exhibited by the different quantities that characterize a giant caloric effect i.e. isothermal entropy change, adiabatic temperature change and $R C$.

It is found that the BCE is larger for samples transforming martensitically above the Curie point of the austenite. As the martensitic transition occurs below the Curie point, all quantities start to decrease. Such a decrease is due to the lowering of the transition entropy change, arising from the magnetic contribution to the entropy. With regards to the MCE, the entropy change attains a maximum for those samples with martensitic and magnetic transitions close to each other. However, the adiabatic temperature change and $R C$ are larger for samples transforming martensitically slightly below the Curie point, as a consequence of the stronger sensitivity of the martensitic transition to the magnetic field, resulting from a larger change in magnetization at the transition.

We expect that present findings can provide useful guidelines in the selection of magnetic shape memory alloys for possible refrigerating devices. While it is commonly accepted that a large entropy change is required, for certain specific applications using the MCE it can be more interesting to use materials with slightly lower entropy values but with larger temperature changes and with a broader temperature span of applicability. On the other hand, for BCE applications, weakly magnetic samples exhibit the best performances. It is finally worth mentioning that interesting applications can take advantage of the materials response to several external stimuli. In that case, the opposite trends exhibited by BCE and MCE effects point to a delicate balance between the two effects in order to attain an optimal multicaloric response.

\section{Acknowledgements}

We acknowledge financial support from CICyT (Spain) Projects N. MAT201340590-P and FIS2011-24439, and from the Joint Indo-Spanish project, DG- 
ICyT (Spain) Project No. PRI-PIBIN-2011-0780 and DST (India) Project No. DST/INT/P-39/11. E.S.-T. acknowledges support from AGAUR (Catalonia), and P.L., support from SUR (DEC, Catalonia).

[1] Mañosa L, Planes A, Acet M. J. Mater. Chem. A 2013;1:4925.

[2] Fähler S, Rössler U, Kastner O, Eckert J, Eggeler G, Emmerich H, Entel O, Müller S, Quandt E, Albe K. Adv. Engn. Mater 2012;14:10.

[3] Moya X, Kar-Narayan S, Mathur ND. Nature Mater. 2014;13:439.

[4] Gschneidner KA, Pecharsky VK, Tsokol AO. Rep. Prog. Phys. 2005;68:1479.

[5] Smith A, Bahl C R H, Bjork R, Engelbrecht K, Nielsen K K, Pryds N. Adv. Energy Mat. 2012;2:1288.

[6] Mischenko AS, Zhang Q, Scott JF, Whatmore RW, Mathur ND. Science 2006;311:1270.

[7] Moya X, Stern-Taulats E, Crossley S, González-Alonso D, Kar-Narayan S, Planes A, Mañosa L, Mathur ND. Adv. Mater. 2013;25:1360.

[8] Mañosa L, González-Alonso D, Planes A, Bonnot E, Barrio M, Tamarit JL, Aksoy S, Acet M. Nature Mater. 2010;9:478.

[9] Mañosa L, González-Alonso D, Planes A, Barrio M, Tamarit JL, Titov IS, Acet M, Bhattacharyya A, Majumdar S. Nature Comm. 2011;2:595.

[10] Bonnot E, Romero R, Mañosa L, Vives E, Planes A. Phys. Rev. Lett. 2008;100:125901.

[11] Mañosa L, Jarque-Farnos S, Vives E, Planes A. Appl. Phys. Lett. 2013;103:211904.

[12] Entel P, Buchelnikov VD, Khovailo VV, Zayak AT, Adeagbo WA, Gruner ME, Herper HC, Wassermann EF. J. Phys. D. Appl. Phys. 2006;39:865.

[13] Acet M, Mañosa L, Planes A. Handbook of Magnetic Materials 2011;19:231. 
[14] Planes A, Mañosa L, Acet M, J. Phys.:Condens. Matter 2009;21:233201.

[15] Castillo-Villa PO, Soto-Parra DE, Matutes-Aquino JA, Ochoa-Gamboa RA, Planes A, Mañosa L, González-Alonso D, Stipcich M, Romero R, Rios-Jara D, Flores-Zuñiga H. Phys. Rev B 2011;83:174109.

[16] Millán-Solsona R, Stern-Taulats E, Vives E, Planes A, Sharma AJ, Nayak AK, Suresh KG, Mañosa L. Appl. Phys. Lett. 2014;105:241901.

[17] Hu F, Shen B, Sun J. Appl. Phys. Lett 2000;76:3460.

[18] Krenke T, Duman E, Acet M, Wassermann EF, Moya X, Mañosa L, Planes A. Nature Mater. 2005;4:450.

[19] Kihara T, Xu X, Ito W, Kainuma R, Tokunaga M. Phys. Rev. B 2014;90:214409.

[20] Ito W, Imano Y, Kainuma R, Sutou Y, Oikawa K, Ishida K. Metall. and Mater. Trans. A 2007;38A:759.

[21] Kustov S, Corró ML, Pons J, Cesari E. Appl. Phys. Lett 2009;94:191901.

[22] Cui J, Chu YS, Famodu OO, Furuya Y, Hatrick-Simpers J, James RD, Ludwig A, Thienhaus S, Wuttig M, Zhang Z, Takeuchi I. Nature Mater. 2006;5:286

[23] Song Y, Chen X, Dabade V, Shield TW, James RD. Nature 2013;502:85.

[24] Srivastava V, Chen X, James RD. Appl. Phys. Lett. 2010;97:014101.

[25] Stern-Taulats E, Catillo-Villa PO, Mañosa L, Frontera C, Pramanick S, Majumdar S, Planes A. J. Appl. Phys. 2014;115:173907.

[26] Mañosa L, Moya X, Planes A, Gutfleisch O, Lyubina J, Barrio M, Tamarit JL, Aksoy S, Krenke T, Acet M. Appl. Phys. Lett. 2008;92:012515.

[27] Emre B, Yüce S, Stern-Taulats E, Planes A, Fabbrici S, Albertini F, Mañosa L. J. Appl. Phys. 2013;113:213905.

[28] Chen JH, Bruno NM, Karaman I, Huang Y, Li J, Ross JH. J. Appl. Phys. 2014;203901:203901. 
[29] Miyamoto T, Ito W, Umetsu RY, Kainuma R, Kanomata T, Ishida K. Scripta mater. 2010;62:151.

[30] Li CM, Luo HB, Hu QM, Yang R, Johansson B, Vitos L. Phys. Rev. B 2012;86:214205.

[31] Nayak AK, Salazar-Mejia C, D'Souza SWD, Chadov S, Skourski Y, Felser C, Nicklas M. Phys. Rev. B 2014;90:220408(R).

[32] Aksoy S, Acet M, Deen PP, Mañosa L, Planes A. Phys. Rev. B 2009;79:212401.

[33] Mañosa L, Stern-Taulats E, Planes A, Lloveras P, Barrio M, Tamarit JL, Emre B, Yüce S, Fabbrici S, Albertini F. phys. stat. sol. (b) 2014;251:2114.

[34] Yuce S, Barrio M, Emre B, Stern-Taulats E, Planes A, Tamarit JL, Mudryk Y, Gschneidner KA, Pecharsky VK, Mañosa L. Appl. Phys. Lett. 2012;101:071906.

[35] Stern-Taulats E, Planes A, Lloveras P, Barrio M, Tamarit JL, Pramanick S, Majumdar S, Frontera C, Mañosa L. Phys. Rev. B 2014;89:214105.

[36] Matsunami D, Fujita A, Takenaka K, Kano M. Nature Mater. 2014;14:73.

[37] Porcari G, Cugini F, Fabbrici S, Pernechele C, Albertini F, Buzzi M, Mangia M, Solzi M. Phys. Rev. B 2012;86:105532.

[38] Ghorbani Zavareh M, Salazar Mejía C, Nayak AK, Skourski Y, Wosnitza C, Felser C, Nicklas M. Appl. Phys. Lett. 2015;106:071904.

[39] Fukuda T, Kakeshita T, Lee Y. Acta Mater. 2014;81:121.

[40] Ito W, Ito K, Umetsu RY, Kainuma R, Koyama K, Watanabe K, Fujita A, Oikawa K, Ishida K, Kanomata T. Appl. Phys. Lett. 2008;92:021908.

[41] Recarte V, Pérez-Landázabal JI, Sánchez-Alarcos V, Zablotskii V, Cesari E, Kustov S. Acta Mater. 2012;60,3168. 

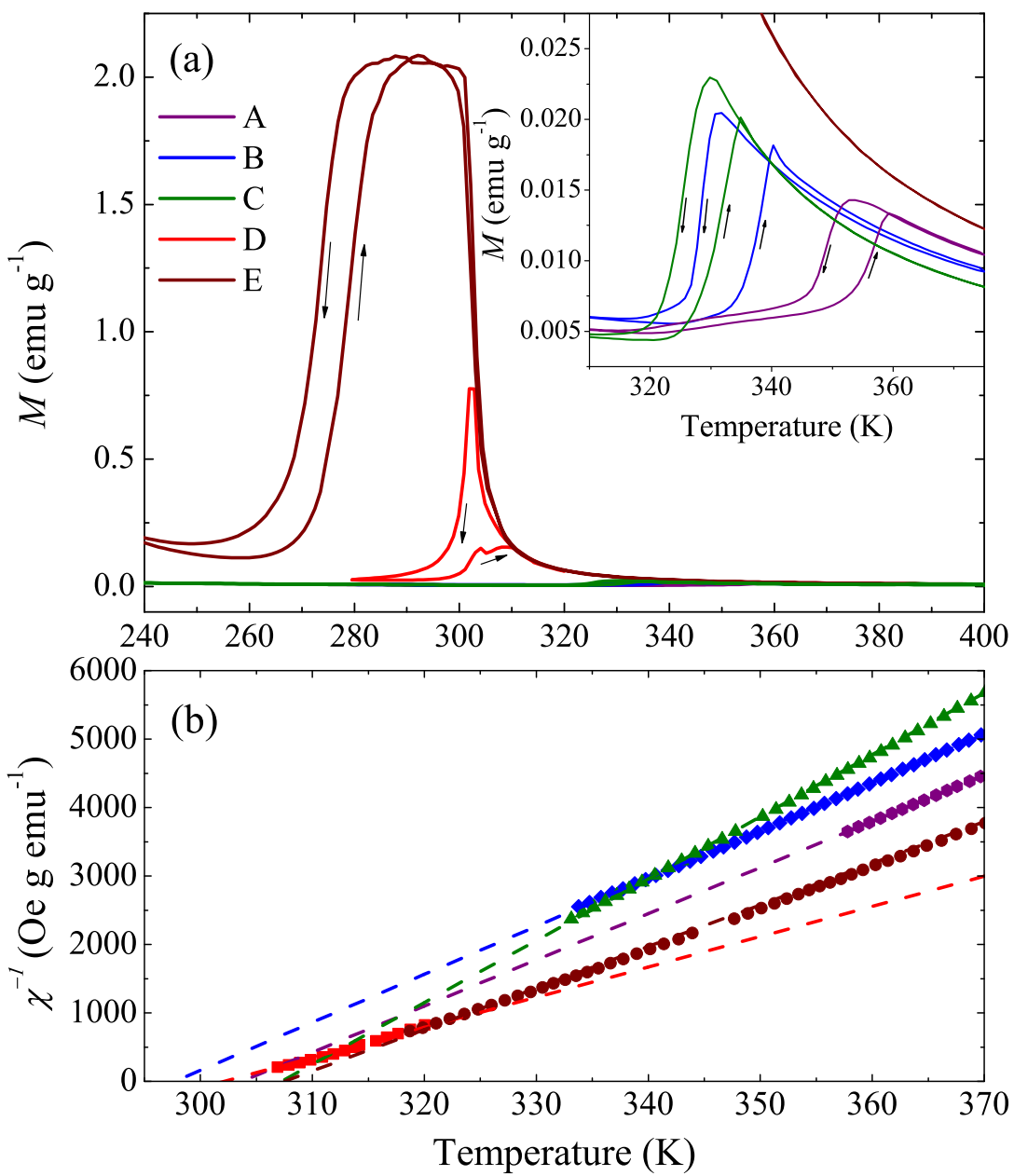

Figure 1: (a) Temperature dependence of the magnetization measured under a magnetic field of 50 Oe. The inset shows an enlarged view over a restricted temperature domain that illustrates the changes taking place at the martensitic transition in the paramagnetic state. The arrows indicate cooling and heating runs. (b) Inverse of the magnetic susceptibility as a function of temperature. Dashed lines are linear fit to the data. 

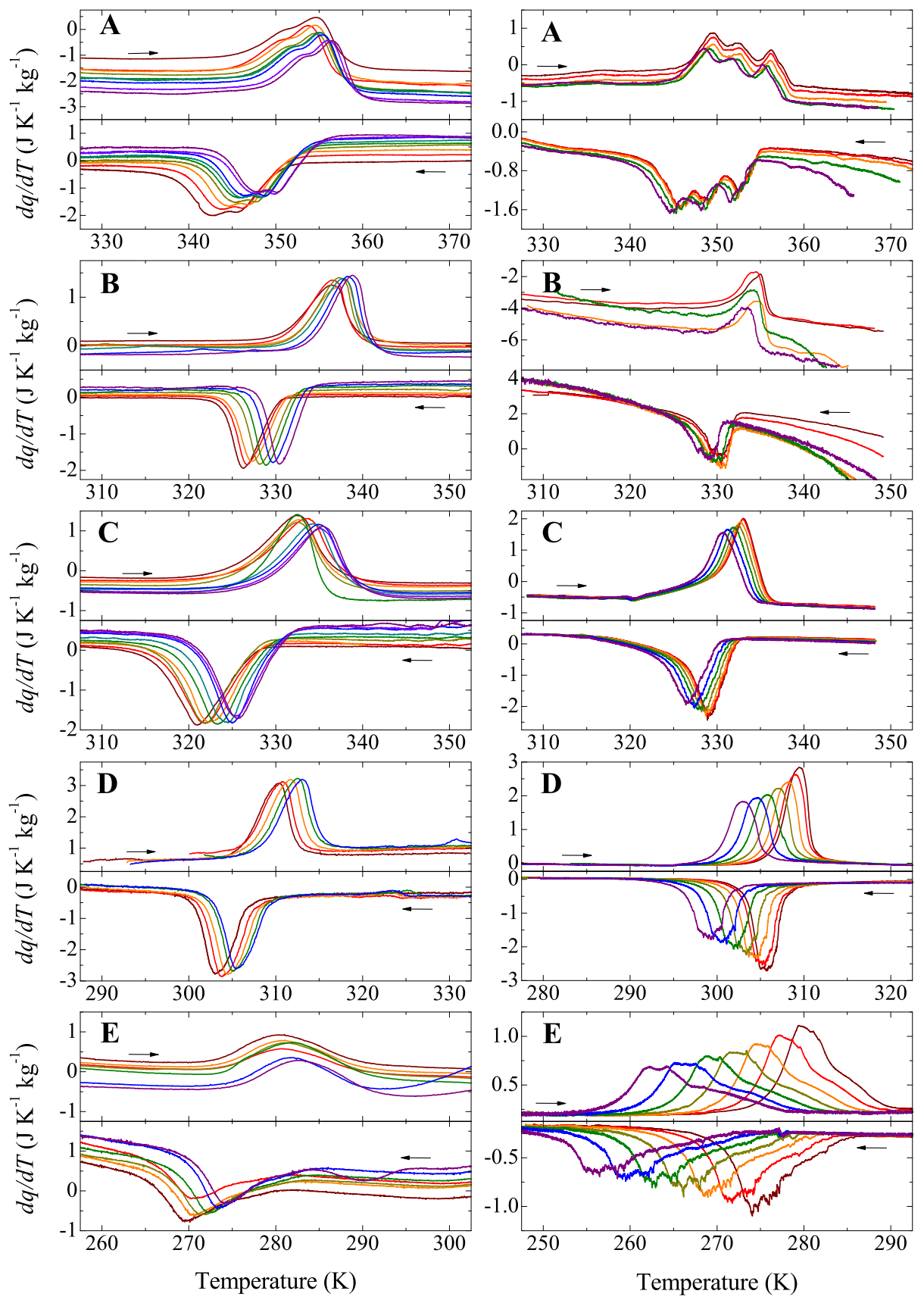

Figure 2: Left panels: Calorimetric curves recorded under hydrostatic pressure. From left to the rigth the curves correspond to the following applied pressures: sample A, $0.3 \mathrm{kbar}$, $0.5 \mathrm{kbar}, 0.8 \mathrm{kbar}, 1.0 \mathrm{kbar}, 1.2 \mathrm{kbar}, 1.5 \mathrm{kbar}, 1.7 \mathrm{kbar}, 2.2 \mathrm{kbar}$ and $2.5 \mathrm{kbar}$. Sample B, 0 kbar, 0.3 kbar, 0.6 kbar, 1.1 kbar, $1.6 \mathrm{kbar}, 2.0 \mathrm{kbar}$ and $2.6 \mathrm{kbar}$. Sample C, $0 \mathrm{kbar}$, $0.3 \mathrm{kbar}, 0.5 \mathrm{kbar}, 0.8 \mathrm{kbar}, 1.0 \mathrm{kbar}, 1.6 \mathrm{kbar}, 2.1 \mathrm{kbar}, 2.3 \mathrm{kbar}$ and $2.5 \mathrm{kbar}$. Sample D, 0 kbar, 0.6 kbar, 1.1 kbar, 1.6 kbar and 2.8 kbar. Sample E, 0 kbar, 0.3 kbar, 0.5 kbar, $0.8 \mathrm{kbar}, 1.0 \mathrm{kbar}, 1.4 \mathrm{kbar}, 2.0 \mathrm{kbar}$ and 2.5. Right panels: Calorimetric curves recorded under magnetic field. From right to the left the curves correspond to magnetic fields of 0,1,2,3,4 and $6 \mathrm{~T}$ (samples $\mathrm{A}$ and $\mathrm{B}$ ) and 0,1,2,3,4,5 and $6 \mathrm{~T}$ (samples C,D and E). In each panel, upper curves (endothermal process) correspond to heating runs while bottom curves (exothermal process) correspond to cooling runs. 


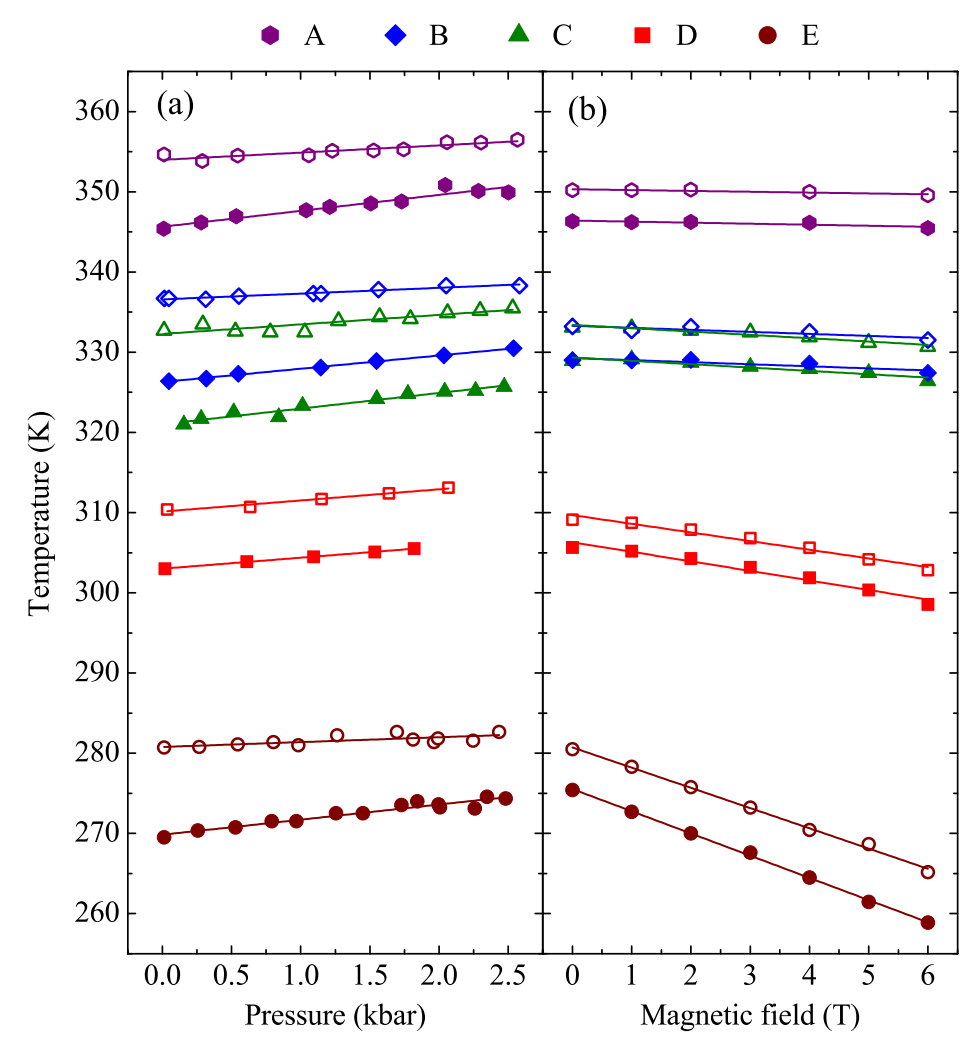

Figure 3: Transition temperatures for the forward (solid symbols) and reverse (open symbols) martensitic transitions, as a function of hydrostatic pressure (a) and (b) magnetic field. Lines are linear fit to the data. 

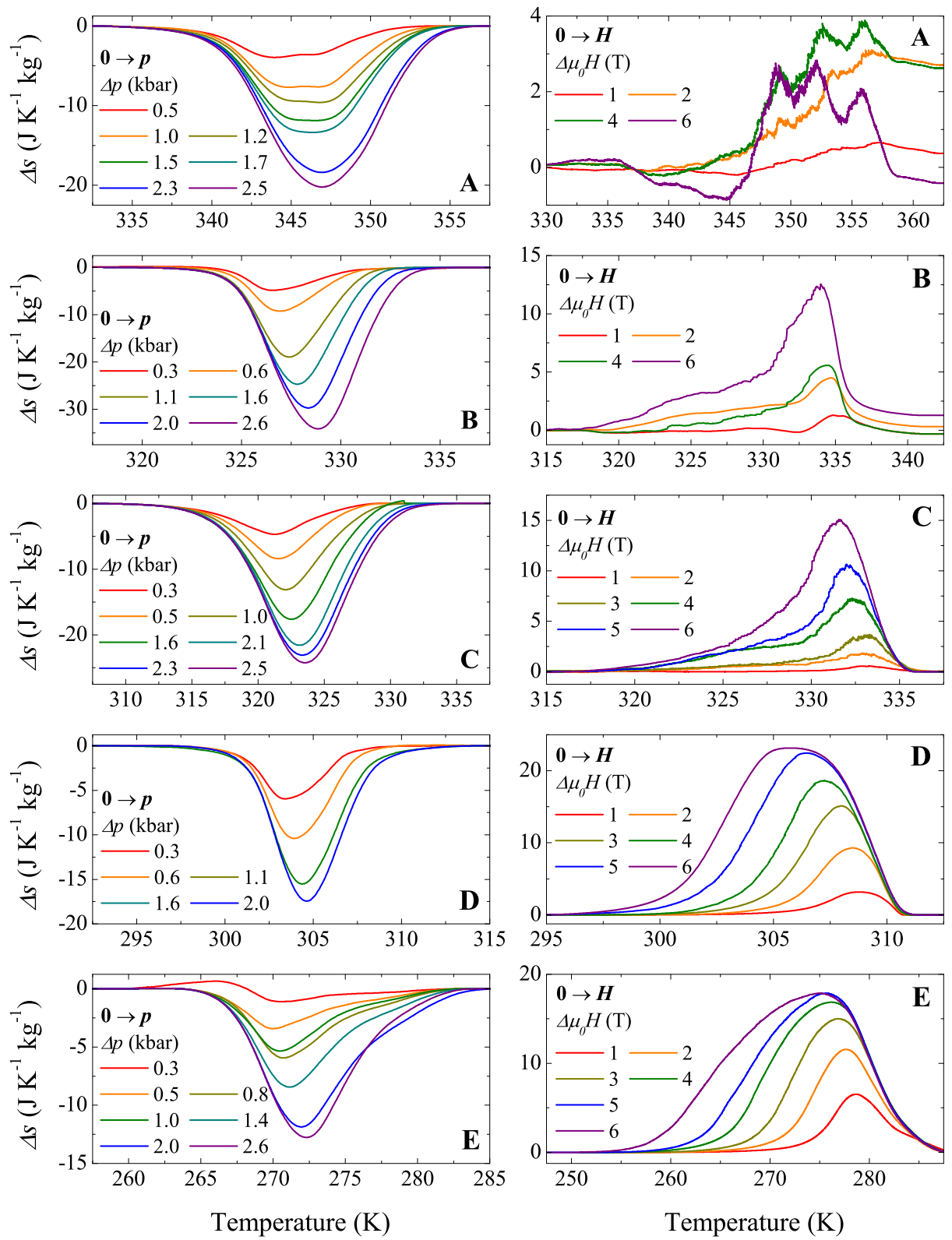

Figure 4: Isothermal entropy changes associated with the barocaloric (left panels) and magnetocaloric (right panels) effects. Barocaloric data are computed from calorimetric curves under hydrostatic pressure for cooling runs and magnetocaloric data, from calorimetric curves under magnetic field for heating runs. 

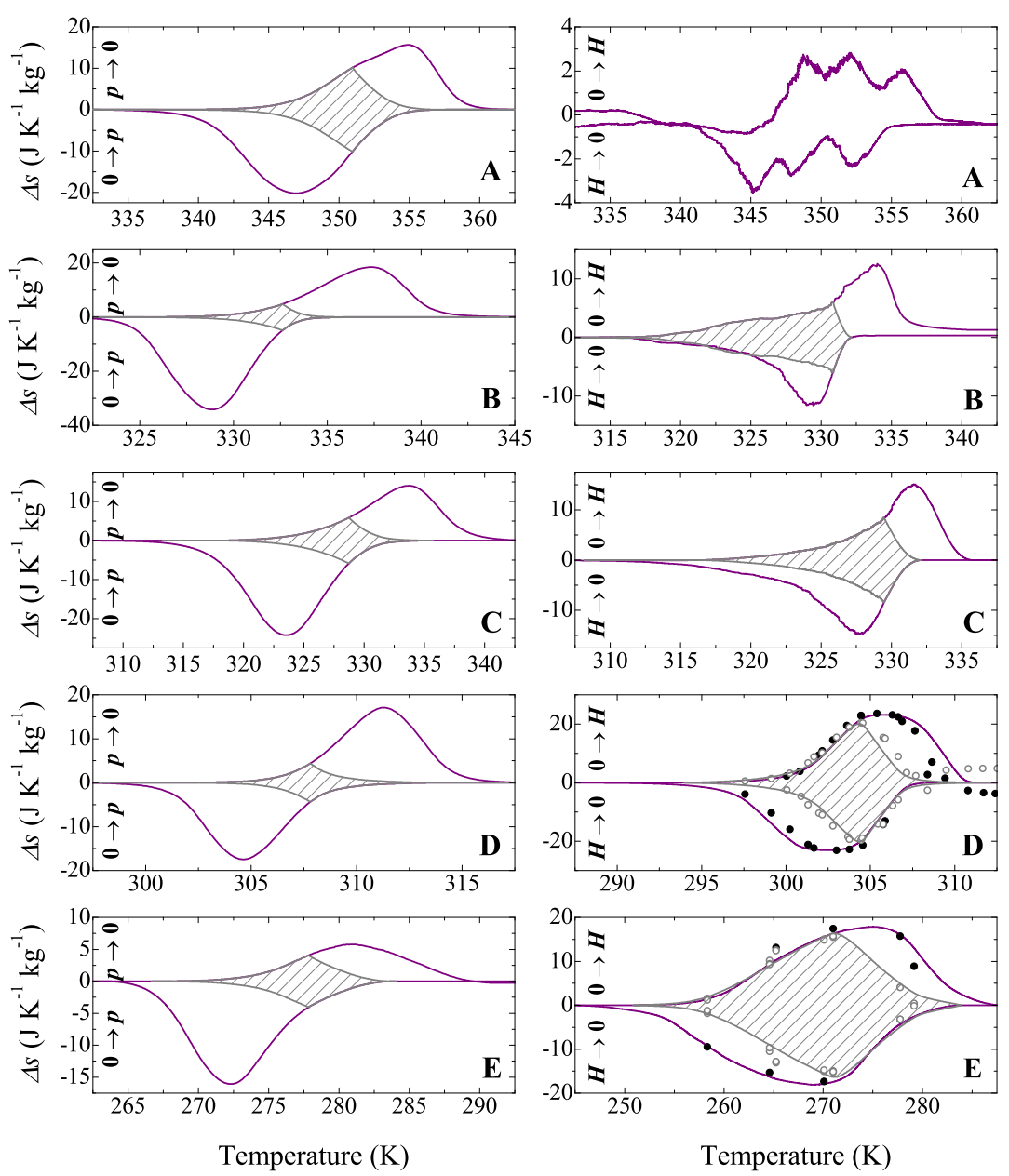

Figure 5: Maximum isothermal entropy change as a function of hydrostatic pressure (a,barocaloric effect) and as a function of magnetic field (b, magnetocaloric effect). 


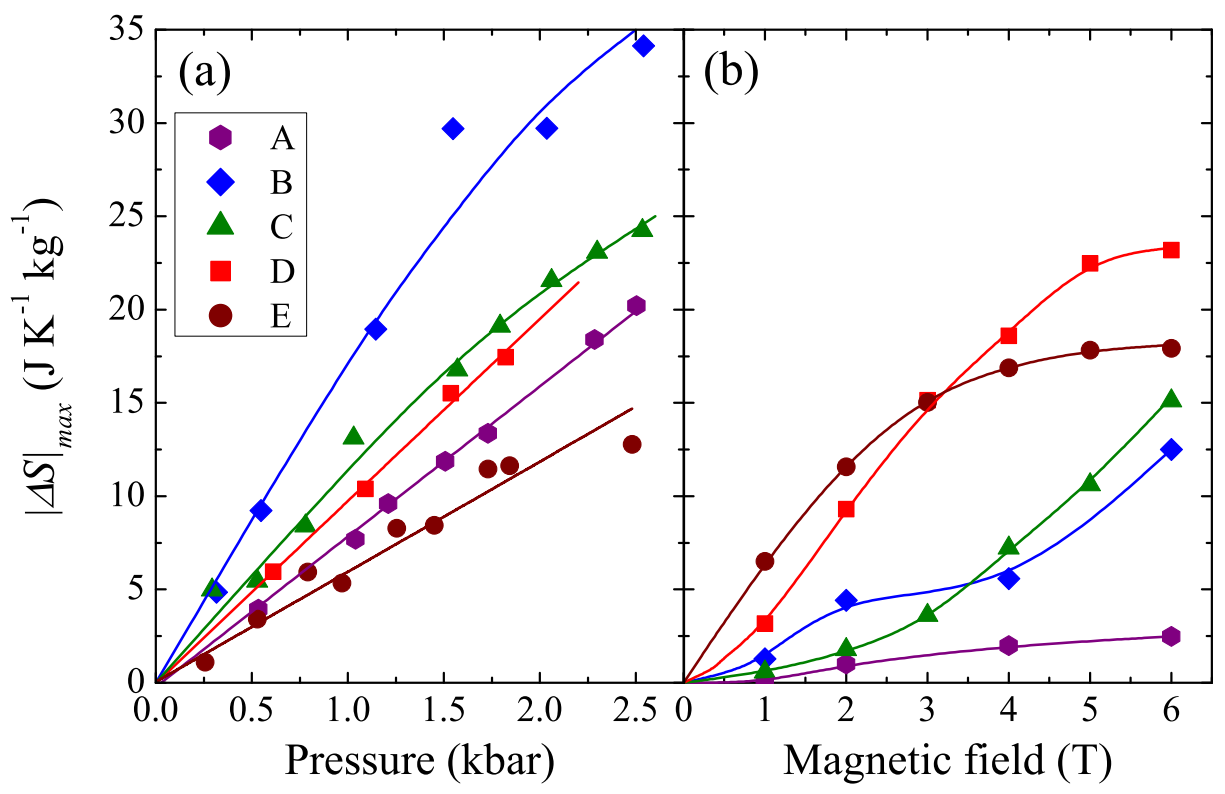

Figure 6: Left panels: Isothermal entropy changes associated with the barocaloric effect for a hydrostatic pressure of $2.5 \mathrm{kbar}$ (except for sample D which corresponds to $2 \mathrm{kbar}$ ). Right panels: Isothermal entropy values associated with the magnetocaloric effect for a magnetic field of $6 \mathrm{~T}$. Purple lines correspond to the values obtained by the quasi-direct method. Symbols correspond to direct isothermal measurements (sweeping magnetic field) where solid circles stand for the first application (and removal) of the magnetic field and open circles, for successive field cycling. The reversibility region is indicated by the shaded area. 


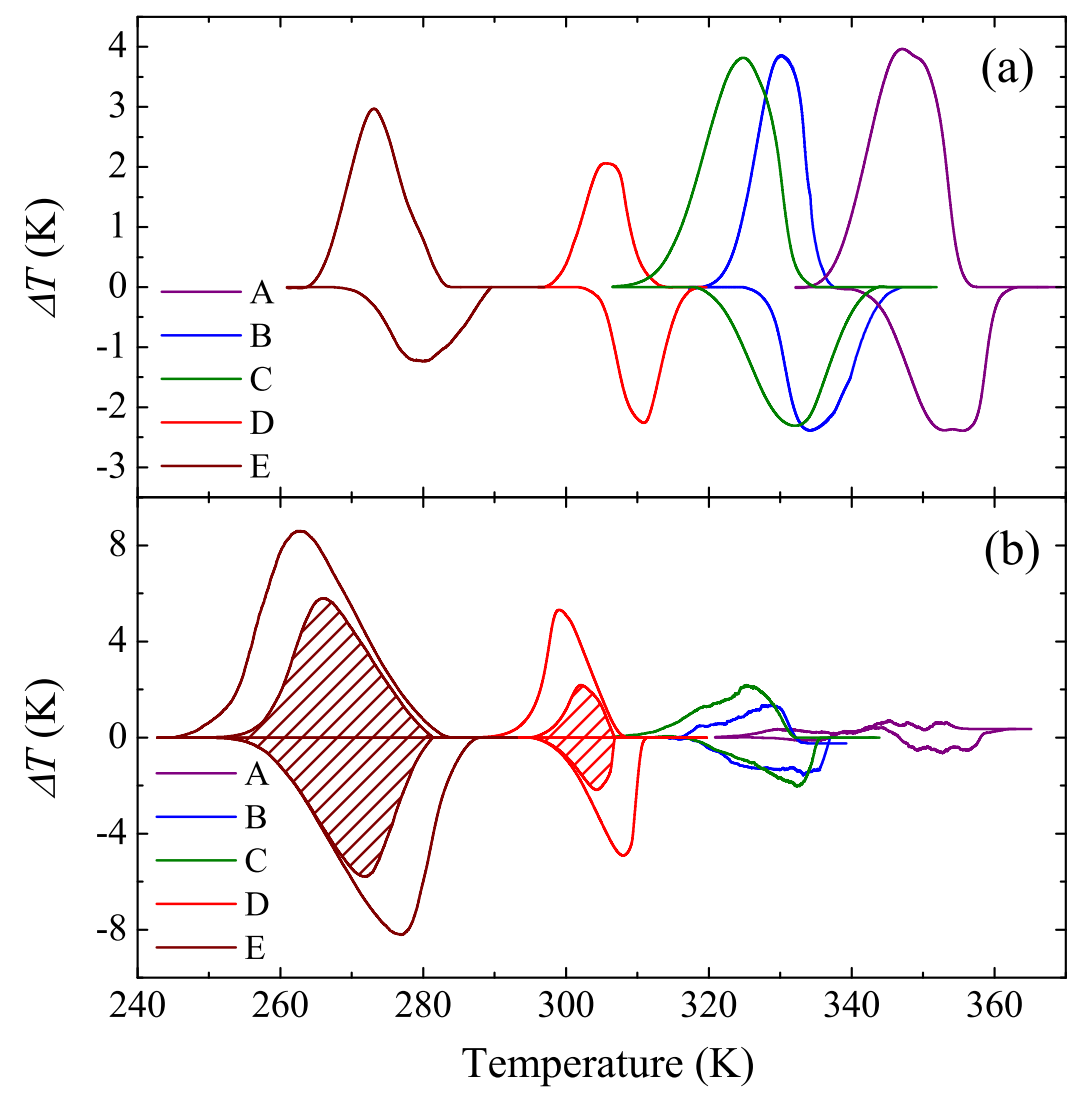

Figure 7: (a) Adiabatic temperature changes corresponding to the application (upper curves) and removal (lower curves) of a hydrostatic pressure of $2.5 \mathrm{kbar}$ (except for sample $\mathrm{D}$ that corresponds to $2 \mathrm{kbar}$ ). (b) Adiabatic temperature changes corresponding to the application (lower curves) and removal (upper curves) of a magnetic field of $6 \mathrm{~T}$. Shaded areas indicate the reversibility region. 

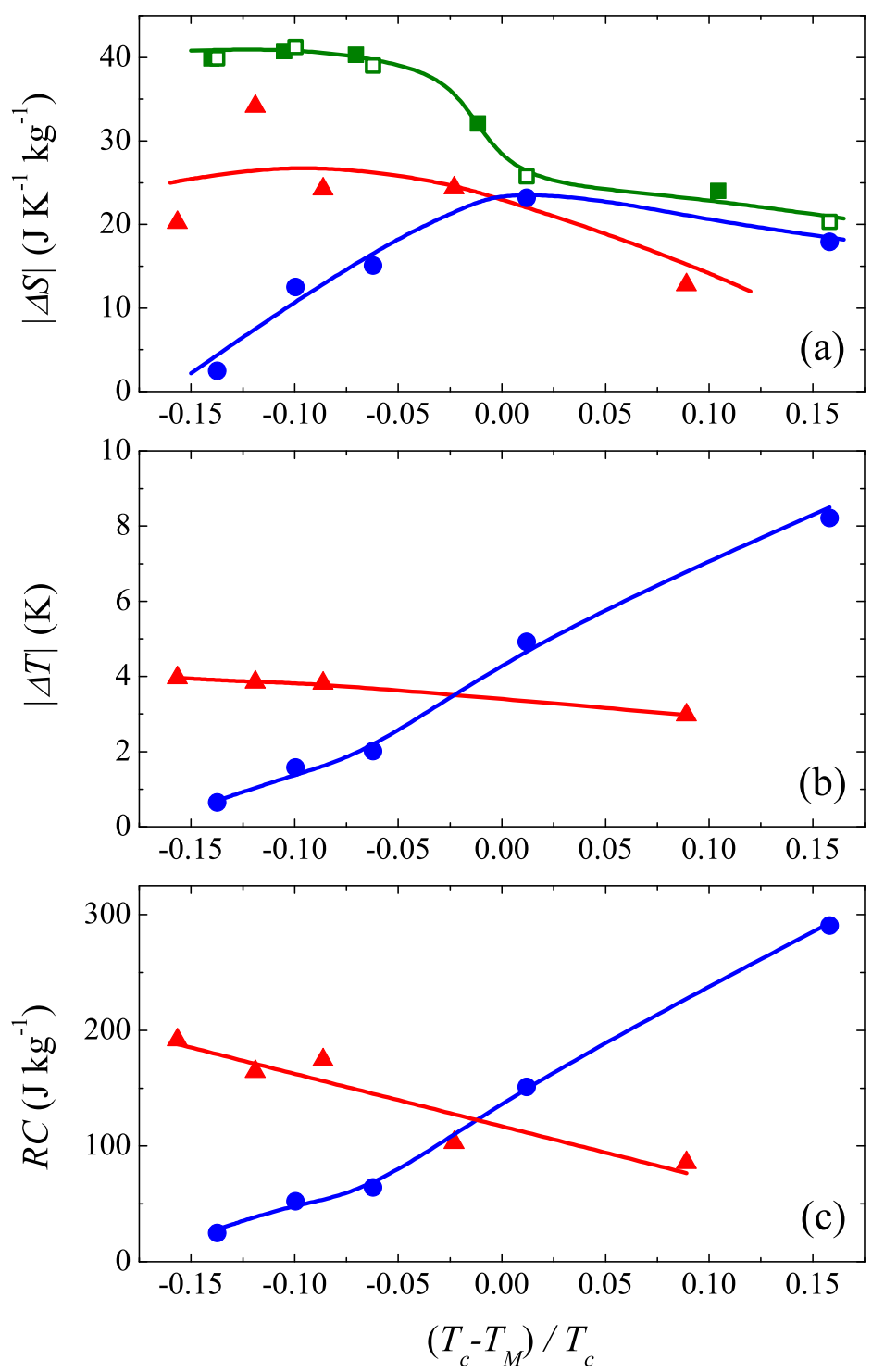

Figure 8: (a) Transition entropy change at zero field and atmospheric pressure (solid green squares) and at $6 \mathrm{~T}$ and atmospheric pressure (open green squares); pressure induced entropy change (red triangles, barocaloric effect) and magnetic field induced entropy change (blue circles, magnetocaloric effect) as a function of the reduced temperature (b) Adiabatic temperature change for the barocaloric (red triangles) and magnetocaloric (blue circles) effects as a function of the reduced temperature. (c) Refrigerant capacity for the barocaloric (red triangles) and magnetocaloric (blue circles) effects as a function of the reduced temperature. For all samples $p=2.5 \mathrm{kbar}$ and $\mu_{0} H=6 \mathrm{~T}$. The entropy and $R C$ values at 2.5 kbar for sample $\mathrm{D}$ have been estimated from extrapolation of the data at lower pressures. Lines are guides to the eye. 